\title{
Proton beam radiotherapy in the management of uveal melanoma: Clinical experience in Scotland
}

\author{
Samir Aziz' \\ Alex Taylor' \\ Andrzej McConnachie ${ }^{3}$ \\ Alex Kacperek ${ }^{2}$ \\ Ewan Kemp' \\ 'Tennent Institute of Ophthalmology, \\ Gartnavel General Hospital, Glasgow, \\ Scotland, UK; ${ }^{2}$ Clatterbridge Centre \\ for Oncology, Wirral, England, UK; \\ ${ }^{3}$ Robertson Centre for Biostatistics, \\ University of Glasgow, Glasgow, \\ Scotland, UK
}

\begin{abstract}
Aim: To evaluate proton-beam radiotherapy (PBRT) in the management of uveal melanoma in Scotland.

Methods: A retrospective review was undertaken on all patients receiving PBRT for uveal melanoma (1994-2005). Data obtained included: gender, past ocular/medical history, age, presenting complaint(s), diagnosis, laterality, tumor location/ultrasound characteristics, visual acuity (VA) and intraocular pressure. At post-treatment reviews (3, 6, 12, and 24 months), the following data was obtained: VA, intraocular pressure, tumor appearance and ultrasound characteristics. Mean follow up was 38.8 months.

Results: Seventy-six patients were included. Mean age was 64 years; male to female ratio was 1.1:1. Ninety-seven percent demonstrated initial treatment response; $87 \%$ had successful control of tumor growth. Mean pre-treatment tumor height was $6.2 \mathrm{~mm}$ v.s. $4.8 \mathrm{~mm}$ post-irradiation ( $p<0.001$ ). Pre-irradiation VA was $<3 / 60$ in $18.5 \%$ compared with $74 \%$ post-irradiation $(\mathrm{p}<0.0001)$. There was a statistically significant association between adverse events (enucleation, metastasis) and greater maximal basal tumor diameter. Eighteen eyes were enucleated. The median survival time was estimated to be 54 months.

Conclusion: In our experience, PBRT is a precise, reliable and effective treatment in the management of large, and previously treated uveal melanomas. It prevents enucleation in the majority at short term follow-up.
\end{abstract}

Keywords: proton-beam radiotherapy, uveal melanoma, visual acuity, enucleation, tumor

\section{Introduction}

Uveal melanoma is the most common primary adult ocular malignancy ${ }^{1-5}$ with an incidence of $0.6 / 100,000 .{ }^{1}$ It is the only potentially fatal intraocular tumor in the adult. ${ }^{2}$

The standard treatment of choroidal melanoma used to be primary enucleation. ${ }^{4-6}$ Zimmerman 7 in the 1970 's suggested this may not be the best management. Globe preserving management is now favored. Such techniques include: laser photocoagulation, ${ }^{1,8,9}$ transpupillary thermotherapy, ${ }^{1,8,10}$ local resection, radiotherapy (charged particle or brachytherapy) and, most recently, the gamma knife. ${ }^{11,12}$ Enucleation is reserved for an end stage disease.

Proton-beam radiotherapy (PBRT) was first utilized in the management of uveal melanoma in $1975 .^{13}$ It is now predominantly used for choroidal and ciliary body melanomas. Ocular complications may arise, ${ }^{2,5,14}$ such as radiation retinopathy, neuropathy and cataract. ${ }^{2,14}$ They are largely dependent on tumor size and location. ${ }^{2}$ This retrospective study was performed to evaluate the use of PBRT in the management of relatively large uveal melanoma and to review our treatment experience.

\section{Methods}

A retrospective case note review was performed on 76 patients who received PBRT for uveal melanoma. The diagnosis was established utilizing clinical examination features, 
photographs, and ultrasound findings. Histopathological confirmation was performed on all enucleation specimens. All patients were jointly managed at the Tennent Institute of Ophthalmology, Glasgow, Scotland (referral centre) and the Clatterbridge Centre for Oncology, Wirral, England (treatment centre).

Initially, some lesions underwent photocoagulation, local resection (medium sized equatorial lesions), or brachytherapy (maximal vertical tumor height [MVTH] of $<5 \mathrm{~mm}$ ) when they proved to be amenable to such modality of treatment. Multiple treatment failure was managed with enucleation. Patients were offered PBRT if their lesions were unsuitable for alternative treatments, if they demonstrated prior treatment failure or had iris/ciliary body tumors.

All patients were assessed by the same consultant ophthalmologist (EGK) and had ultrasound examinations performed by three consultant radiologists. All patients had irradiation planning and treatment delivered by the same multidisciplinary team.

\section{PBRT protocol}

Stage one involved surgical placement of tantalum clips to demarcate the tumor periphery. Stage two incorporated the simulation and planning of patient specific treatment. Cranial X-rays, fundus photographs, and ocular ultrasound data were utilized to create computer-generated patient eye/tumor models. Clip positions were used at simulation to plan individualized irradiation fields. Stage three involved delivery of PBRT in four consecutive, daily fractions (30 seconds each) to a total dose of 53.1 protons Gray (58 Gy Co-60 equivalents).

The following data were obtained for each patient: gender, past ocular/medical history, age at presentation, presenting complaint(s), ocular diagnosis, laterality, anatomical tumor location, tumor ultrasound characteristics [(MVTH) and maximal basal tumor diameter (MBTD)], best corrected visual acuity (BCVA), intraocular pressure (IOP) and PBRT data (total dose, duration, number of fractions, maximum exposure dose to various ocular structures). At post-treatment review (3, 6, 12, and 24 months), the following data was obtained: BCVA, IOP, tumor appearance (clinical examination, fundus photography) and ultrasound characteristics. The development of metastatic disease and patient mortality was documented. Tumor growth was monitored utilizing serial ultrasound examinations, clinical examination, and fundus photography. Treatment endpoints included evidence of tumor growth, enucleation, and patient death. The follow-up period was calculated from first treatment date until documentation of tumor growth, enucleation, or last clinic visit.

Statistical analysis was performed using p-value for assessment of statistical significance of tumor dimensions and changes in BCVA. Kaplan-Meier survival analysis was applied for assessment of the cumulative adverse event rate over time. The effects of baseline characteristics on subsequent adverse event rates were estimated using Cox proportion hazards models.

\section{Results}

\section{Demographics}

Seventy-six patients received PBRT for uveal melanoma between January 1994 and June 2005 (Table 1). Seventy had a clinical diagnosis of choroidal melanoma and six had presumed iris/ciliary body melanoma. Nine patients had previously received treatment: Ruthenium-106 plaque $(n=4)$, local tumor resection $(\mathrm{n}=2)$, and laser photocoagulation $(n=3)$. Patients were followed up for a mean of 38.8 months (range 3-122).

\section{Tumor growth}

Seventy-four patients (97\%) demonstrated an initial response to treatment (stabilization of tumor growth, with or without regression). There was evidence of continued tumor growth

Table I Baseline characteristics

\begin{tabular}{lll}
\hline Age at treatment (years) & Median (Range) & $\mathbf{6 4}(\mathbf{2 9 - 8 8})$ \\
\hline Sex & Male & $40(53 \%)$ \\
& Female & $36(47 \%)$ \\
Affected eye & Right & $40(53 \%)$ \\
Presenting symptom(s) & Left & $36(47 \%)$ \\
& Blurred vision & $35(46 \%)$ \\
& Asymptomatic & $22(28.9 \%)$ \\
& Flashes and/or floaters & $10(13.2 \%)$ \\
& Visual field defect & $5(6.6 \%)$ \\
Tumor location & Others & $4(5.3 \%)$ \\
& Choroidal & 70 \\
& Straddling equator & $45(59 \%)$ \\
& Posterior to equator & $25(33 \%)$ \\
Subretinal fluid & Iris/ciliary body & 6 \\
& Present & $50(66 \%)$ \\
Referral source & Absent & $26(34 \%)$ \\
& Optometrist & $32(42 \%)$ \\
& Ophthalmologist & $30(39 \%)$ \\
& GP & $2(3 \%)$ \\
& Unknown & $12(16 \%)$ \\
\hline
\end{tabular}

Note: Number of patients (percentage of total) unless stated otherwise. 
$<2$ months post treatment in both remaining patients, one with extension along the optic nerve. Sixty-six (87\%) patients had successful control of tumor growth throughout the entire follow-up period. Ten (13\%) demonstrated evidence of long-term treatment failure (no response to treatment [ $n=2]$, delayed tumor progression [ $\mathrm{n}=4$ ], development of metastatic disease [ $n=4]$, of which one patient died during follow-up). Three (out of 10) demonstrated both tumor progression and metastatic disease.

\section{Pre- and post-irradiation characteristics}

In patients for whom both ultrasound measurements were available, there was a statistically significant reduction in MVTH post treatment $(\mathrm{N}=57$; mean change, $-1.65 \mathrm{~mm}$; $\mathrm{p}<0.001)$ (Table 2). Fifty-one percent demonstrated a $1.5 \mathrm{~mm}$ decrease in height by one and a half years post-treatment. A reduced MBTD was also observed post-treatment, without reaching statistical significance $(\mathrm{N}=70$; mean change, $-0.52 \mathrm{~mm} ; \mathrm{p}=0.072)$.

\section{Visual acuity}

Fourteen patients (18.5\%) had a BCVA of $<3 / 60$ prior to radiotherapy compared with $56(74 \%)$ afterwards $(\mathrm{p}<0.0001)$ (Table 2). Seven patients showed post-treatment improvement in BCVA. Tumors in these cases were located inferotemporally $(n=5)$, superonasally $(n=1)$, and at the posterior pole in the remaining patient. In this improved BCVA subgroup, the mean (SD) pre treatment MBTD was $14 \mathrm{~mm}(2.4 \mathrm{~mm})$ which reduced to $12.4 \mathrm{~mm}(1.2 \mathrm{~mm})$ by the last visit. The mean (SD) MVTH was $6.0 \mathrm{~mm}(2.0 \mathrm{~mm})$ and reduced to $4.4 \mathrm{~mm}(1.8 \mathrm{~mm})$ by the last visit. Reduction in vision is mainly due to complications of treatment (42 patients, $55.2 \%$ ).

\section{Complications}

The most common complication following PBRT was associated retinopathy. Rubeosis iridis was the second most common followed by cataract (Table 3 ).

\section{Enucleation and metastasis}

Eighteen patients $(24 \%, 11$ were male) underwent enucleation during the follow-up period, of which 13 had associated retinal detachment. Indications for enucleation were: local tumor recurrence $(n=5)$, refractory glaucoma $(n=12)$, and optic nerve involvement $(n=1)$. Two patients had received prior treatment (laser and local resection). Enucleation was performed on average 22.3 months post-treatment.

Seven patients (four were male) developed clinically evident distant metastases during follow-up. One died from their disease during this period and one had histopathology confirmed disease (liver biopsy). Three presented with clinical evidence of distant metastases 11, 15, and 16 months post enucleation, respectively; two of these three patients showed initial tumor treatment response, the third did not. The remaining two patients developed clinically evident metastatic disease 25 and 31 months post-PBRT, respectively.

The mean age at diagnosis of metastasis was 60 years. The mean (SD) MBTD for this subgroup was $12.2 \mathrm{~mm}(2.8 \mathrm{~mm})$ and MVTH was $6.2 \mathrm{~mm}$ (1.9 mm). All had hepatic involvement; two also demonstrated breast and spleen metastases. Six had accompanying secondary retinal detachment prior to PBRT.

The duration from treatment date to development of first adverse event (enucleation or late metastatic disease) or the end of follow-up was calculated for every patient; Figure 1 shows the Kaplan-Meier estimate of the cumulative adverse event rate over time. The estimated survival at the maximal

Table 2 Pre- and post-irradiation clinical characteristics

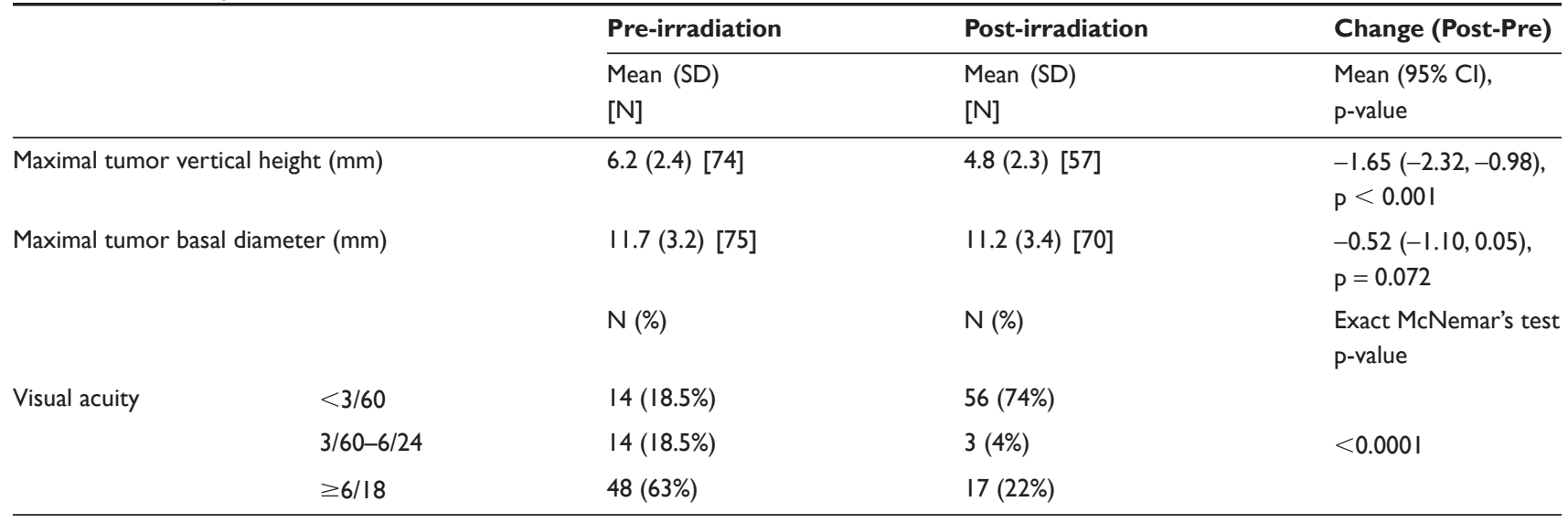

Note: Mean changes not necessarily equal to differences between mean values, due to missing data. Abbreviations: N, number of patients; SD, standard deviation. 
Table 3 Ocular complications of proton beam irradiation (multiple in some patients)

\begin{tabular}{ll}
\hline Complication & $\begin{array}{l}\text { Number of patients } \\
\text { (\% of total) }\end{array}$ \\
\hline Retinopathy & $30(39 \%)$ \\
Rubeosis iridis & $16(21 \%)$ \\
Cataract & $15(20 \%)$ \\
Uveitis & $13(17 \%)$ \\
Optic neuropathy & $12(16 \%)$ \\
Maculopathy & $3(4 \%)$ \\
Keratopathy & $1(1 \%)$ \\
\hline
\end{tabular}

follow-up time was in excess of $50 \%$, so that the median time until the first adverse event could not be estimated from these data. However, the lower 95\% confidence boundary for the median survival time was estimated to be 54 months.

The effects of baseline characteristics on subsequent adverse event rates were estimated using Cox proportion hazards models. The results are presented in Table 4 . The only association observed was an increased risk of adverse events in those with greater MBTD, with an estimated $14.1 \%$ increased hazard (95\% CI 0.3-29.8, $\mathrm{p}=0.045)$ for every $1 \mathrm{~mm}$ increase in basal diameter. This finding must be viewed with some caution, since several variables were tested in this way, and the observed association is of borderline statistical significance.

\section{Resolution of pre-treatment secondary retinal detachment}

Secondary retinal detachment was present in 50 patients (66\%) prior to irradiation treatment. All resolved during follow up, $46 \%$ within the first 3 months. Resolution of the detachment was noted prior to tumor regression.

\section{Discussion}

Proton beam irradiation treatment for uveal melanoma offers several advantages over other methods. ${ }^{6,15}$ It is a precise and reliable technique which achieves excellent tumor-normal tissue dose ratio. ${ }^{6,8,14-16}$ The density of ionization of protons increases markedly near the end of their path (Bragg peak). This characteristic enables accurate treatment, especially important for lesions close to vital ocular structures. ${ }^{6,14,16}$ No handling of radioactive material is required by ophthalmologists dealing with PBRT, in contrast to brachytherapy where handling is required. ${ }^{8}$ External beam radiotherapy with photons will always result in less favorable dose distribution than PBRT. ${ }^{17}$ It appears that the use of gamma-knives leads to higher enucleation rate. ${ }^{18}$

\section{Demographics}

The average patient age in this study population was slightly higher than other reports. ${ }^{6,10,19-21}$ Age however, was not found to be associated with treatment failure in our study $(\mathrm{P}=0.47)$ and by others. ${ }^{8}$

Women with uveal melanoma have previously been found to have more favorable outcomes than men. ${ }^{8}$ This was not duplicated in our study, possibly due to relatively limited follow up time.

\section{Growth}

Local control of disease is defined as clinical evidence of cessation of tumor growth or evidence of tumor regression. ${ }^{4}$

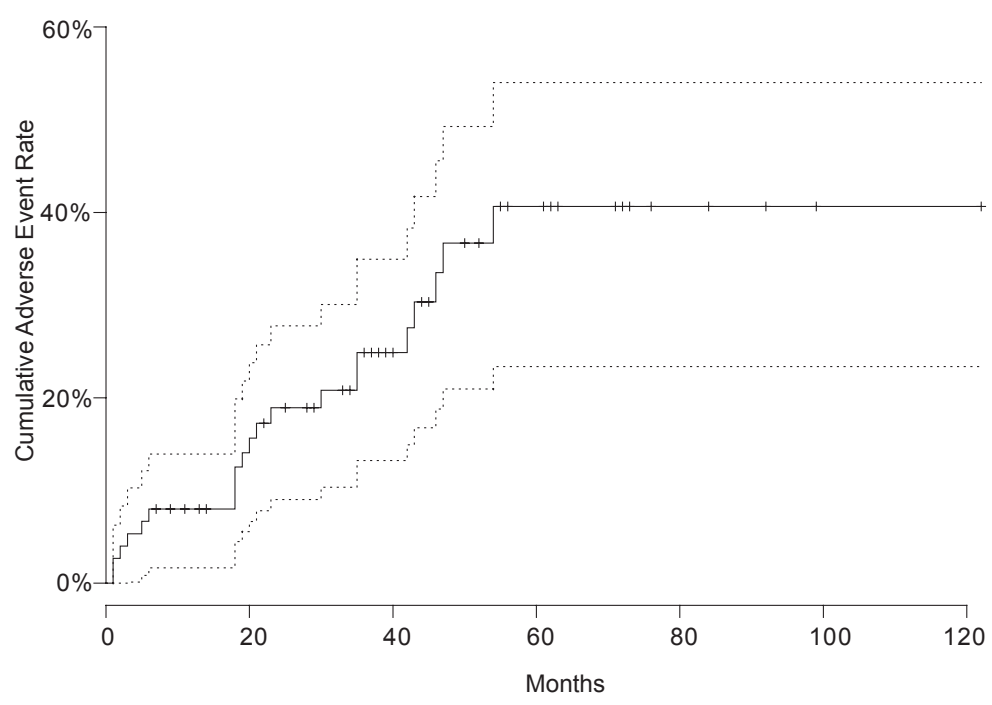

Figure I Kaplan-Meier estimated cumulative adverse event rate (enucleation or distant metastasis) compared with follow-up time after proton beam irradiation. 
In this cohort, $87 \%$ demonstrated this following PBRT. Ultrasound examination revealed a statistically significant decrease $(p<0.001)$ in MVTH following treatment in the last visit. Other studies have shown less dramatic changes in tumor dimensions post-treatment. ${ }^{22}$ The degree of regression response did not appear to be related to initial tumor size in this study. This finding is similar to previous reports. ${ }^{19}$ Most of the treated tumors showed complete or partial regression after the first 6 months of treatment, with a usual range between 1 and 24 months. ${ }^{19}$

Proton beam radiotherapy is an ocular-conserving option that may be considered for the treatment of extra-large uveal melanoma in carefully selected patients. ${ }^{17,21}$

\section{Visual acuity}

Visual acuity deteriorated post-treatment in most patients. Almost two thirds had a pre-treatment BCVA of $\geq 6 / 18$ compared with almost three quarters having BCVA of $<3 / 60$ post-treatment, ( $\mathrm{P}<0.0001$, McNemar's test). This was most likely due to direct radiation damage of vital visual structures. This level of acuity still enables spatial awareness perception, otherwise not present in enucleation cases. Other studies have shown higher levels of retained BCVA post-PBRT. ${ }^{4,10,23}$ It is difficult to compare study outcomes due to variable pre-irradiation VA levels, anatomical sites of the tumors, irradiation doses, and variable complications of treatment.

Male gender has been associated with poorer final BCVA. ${ }^{5}$ This result was not reproduced in our study.

\section{Complications}

Thirty nine percent of our study population developed radiation retinopathy, higher than previously reported. ${ }^{15,21,24}$
Proton beam-induced retinal ischemia predisposes to rubeosis iridis. Twenty one percent of our population developed this complication, Conway and colleagues showed higher rates. ${ }^{21}$ Rubeosis iridis resulting in secondary refractory glaucoma was the most common indication for enucleation in our study, this finding is similar to other reports in the literature. ${ }^{14}$ Tumor-induced angiogenic factors, inflammation secondary to necrosis of the melanoma, retinal detachment, and ischemic retina and iris from irradiation are possible stimuli. ${ }^{25}$ The presence of subretinal fluid was not a significant risk factor in this study for the development of neovascular glaucoma. Similar findings have been reported by Kim and colleagues. ${ }^{26}$ More recently Gragoudas and Lane found that larger tumor volume is the most significant factor associated with iris neovascularisation. ${ }^{27} \mathrm{~A}$ similar finding was reported by Foss. ${ }^{14}$ The early detection of neovascular glaucoma is important in the management of patients with uveal melanoma. ${ }^{28}$ Rubeosis and neovascular glaucoma can be reduced when the anterior segment is spared. ${ }^{25}$ With a very sharp Bragg peak of proton beam, however the irradiation of the anterior segment could be reduced; it resulted in a lower number of anterior segment complications requiring enucleation. $^{29}$

\section{Resolution of pre-treatment secondary retinal detachment}

Secondary retinal detachment resolved clinically in all cases prior to the evidence of tumor regression post-treatment. This finding has been confirmed by a previous study. ${ }^{19}$ Secondary retinal detachments have been documented to develop after PBRT. None of our patients developed this. These have also been shown to resolve completely over time. ${ }^{21}$

Table 4 Estimated effects of baseline characteristics on adverse event rates following treatment with proton beam irradiation-hazard ratios with $95 \%$ confidence intervals and $p$-values

\begin{tabular}{llll}
\hline Characteristic & Effect & Hazard ratio (95\% Cl) & P-value \\
\hline Age & $\geq 60$ vs $<60$ years & $0.7 I(0.27,1.83)$ & 0.47 \\
& $/ 10$ years (linear trend) & $1.16(0.80,1.67)$ & 0.43 \\
Sex & Male vs female & $1.21(0.5 I, 2.88)$ & 0.66 \\
Affected eye & Right vs left & $1.13(0.48,2.67)$ & 0.79 \\
Maximal basal tumor diameter & $>10$ vs $\leq 10 \mathrm{~mm}$ & $1.73(0.67,4.49)$ & 0.26 \\
& $/ \mathrm{mm}$ (linear trend) & $1.14(1.00,1.30)$ & 0.045 \\
Maximal vertical tumor height & $>6$ vs $\leq 6 \mathrm{~mm}$ & $1.54(0.65,3.69)$ & 0.33 \\
& Imm (linear trend) & $1.11(0.94,1.32)$ & 0.22 \\
Subretinal fluid & Present vs absent & $2.01(0.74,5.52)$ & 0.17 \\
\hline
\end{tabular}




\section{Enucleation}

Secondary glaucoma and tumor growth were found to be the leading causes for enucleation following PBRT, both in this study and others. ${ }^{30}$ The post-irradiation enucleation rate in our study $(18 / 76,24 \%)$ was higher than that previously reported by some groups $s^{4,8,14,23,30,31}$ and lower than that reported by others (Table 5). ${ }^{21,24} \mathrm{~A}$ higher probability of enucleation (46\%) has been shown for very large tumors..$^{21,29}$ Enucleation was performed on average 22.3 months posttreatment, which was slightly longer than demonstrated by Kodjikian and colleagues ${ }^{8}$ and shorter than reported by Finger. ${ }^{4}$ The presence of retinal detachment prior to PBRT has been shown to be a significant risk factor for subsequent enucleation. ${ }^{14}$ Our data was consistent with this finding although we had insufficient power to demonstrate this conclusively, with a hazard ratio of $2.01(95 \% \mathrm{CI}$ 0.74-5.52).

Some would argue that enucleation following PBRT is inevitable and that primary enucleation should be advocated instead. However, only a minority of our study population required enucleation. Survival outcome therefore should not be a reason for suggesting enucleation. An increased fractionation scheme or treatment with combination of radiation and angiogenic agents could decrease functional loss and enucleation. ${ }^{25}$

Zehetmayer launched a study using a stereotactic linear accelerator to avoid higher rate of enucleation, which is more convenient for delivery of fractionated treatment. ${ }^{18}$

\section{Metastasis}

Seven patients developed metastases during follow up. Higher and lower rates have been reported (Table 5). ${ }^{8,21,23,24,29,31}$ Maximum tumor vertical height and accompanying retinal detachment have both been established as recognized risk factors for the development of metastatic disease in uveal melanoma. ${ }^{14}$ Our findings support this data.

Despite tumor regression, most melanoma-related metastatic disease manifests within the first five years posttreatment; $15,32,33$ this finding is supported by our finding.

Six patients (7.9\%) showed evidence of local recurrence following PBRT. This rate is in keeping with other literature reports. ${ }^{19}$ Recurrence in our patients was first documented 0.5 to 63 months post-treatment. There are reports demonstrating a lower incidence with earlier recurrence time. ${ }^{8,23,24}$ All six recurrent tumors in this study were large at diagnosis. All but one was located equatorially. The remaining was posteriorly located. These findings are echoed in the literature. ${ }^{8}$

It is difficult to compare reported results referring to various radiation modalities because both dose and fractionation can be very different. ${ }^{31}$ Table 5 showed the least enucleation and metastasis rate in tumors (extra large or located too close to the optic nerve or fovea) treated with noninvasive linear accelerator-based stereotactic irradiation. ${ }^{31}$ However, the prescribed radiation dose is higher than in the current study.

Other fractionation schedules might help to reduce the number of side effects without compromising local control. ${ }^{31}$ It has now become possible to treat small to medium size ocular melanomas using stereotactic radiotherapy with acceptable dose distributions and target localizations. Stereotactic radiotherapy should provide a good alternative to proton therapy. ${ }^{34}$

\section{Conclusions}

Proton beam irradiation treatment was used in this study primarily for the management of large choroidal melanomas and those having failed previous alternative treatment(s). Almost all (97\%) patients demonstrated a response to

Table 5 Local control, metastasis, complications, and visual acuity after proton radiotherapy for uveal melanoma

\begin{tabular}{lllllll}
\hline Author $^{\text {(reference) }}$ & Brovkina $^{24}$ & Gragoudas $^{23}$ & Conway $^{21}$ & Egger $^{29}$ & Dieckmann $^{31}$ & Current study $^{\text {19 }}$ \\
\hline Dose (mean or range in Gy) & $100-125$ & 70 & 56 & 54.5 & $60-70$ & 53.1 \\
Follow-up (mean in months) & 34 & 64 & 28 & 53.2 & $20^{\wedge}$ & 38.8 \\
Recurrence (\%) & 19 & 3 & 33 & 1.9 & 2.2 & 14.5 \\
Enucleation (\%) & 25 & 10 & 46 & 8.2 & 7.7 & 24 \\
Metastasis (\%) & 6.3 & 20 & 10 & $12.3 *$ & 3.3 & 9.2 \\
Cataract (\%) & - & $>42$ & 28.6 & - & 18.9 & 20 \\
Retinopathy (\%) & 5 & - & 9.5 & - & 25.5 & 39 \\
Optic neuropathy (\%) & - & - & 9.5 & - & 20 & 16 \\
Visual acuity (\% $\geq 6 / 60)$ & - & 58 & 25 & - & - & 26.3
\end{tabular}

Notes: *Ocular tumor-related death; ^Median. 
treatment and $87 \%$ showed successful control of tumor growth throughout the entire follow-up period with an associated statistically significant reduction in MVTH post-treatment.

Best corrected visual acuity was markedly reduced (statistically significant) in the majority post treatment although notably those with inferotemporal, superonasal, and posterior pole tumors demonstrated an improvement in BCVA after treatment. Many patients required enucleation (24\%), mainly due to refractory glaucoma. Less than $10 \%$ of patients developed clinically evident distant metastases during follow up, all involving the liver. This study demonstrated a statistically significant association between increased risk of adverse event (enucleation, metastasis) and greater maximal basal tumor diameter.

In our experience, PBRT is a precise, effective, and reliable technique which achieves excellent tumor-normal tissue dose ratio. It is an effective mode (although very expensive $)^{35,36}$ of treatment in the management of large and previously treated choroidal melanomas and is associated with low rates of enucleation over the short term.

\section{Disclosure}

The authors report no conflicts of interest in this work.

\section{References}

1. Chiquet C, Grange JD, Ayzac L, et al. Effects of proton beam irradiation on uveal melanomas: a comparative study of Ki-67 expression in irradiated versus non-irradiated melanomas. Br J Ophthalmol. 2000;84:98-102.

2. Gragoudas ES, Lane AM, Regan S, et al. A randomized controlled trial of varying radiation doses in the treatment of choroidal melanoma. Arch Ophthalmol. 2000;118:773-8.

3. Wright PK, Damato BE. Auditing outcomes after treatment of Scottish patients with uveal melanoma in Liverpool. $J R$ Coll Surg Edinb. 1999;44:260-4.

4. Mindel J, Finger P. Radiation therapy for choroidal melanoma. Therapeutic review. Surv Ophthamol. 1997;42:215-32.

5. Melia BM, Abramson DH, Albert DM, et al. Collaborative Ocular Melanoma Study Group. Collaborative ocular melanoma study (COMS) randomized trial of I-125 brachytherapy for medium choroidal melanoma. I. Visual acuity after 3 years COMS report no. 16. Ophthalmology. 2001;108:348-66.

6. Gragoudas ES, Goitein M, Verhey L, et al. Proton beam irradiation-An alternative to enucleation for intraocular melanomas. Ophthalmology. 1980;87:571-81.

7. Zimmerman LE, McLean IW, Foster WD. Does enucleation of the eye containing a malignant melanoma prevent or accelerate the dissemination of tumour cells. Br J Ophthalmol. 1978;62:420-5.

8. Kodjikian L, Roy P, Rouberol F, et al. Survival after proton-beam irradiation of uveal melanomas. Am J Ophthalmol. 2004;137:1002-10.

9. Fine S. How should we manage a patient with uveal melanoma? Arch Ophthaloml. 1985;103:910-11.

10. Spire M, Devouassoux MS, Kodjikian L, et al. Primary transpupillary thermotherapy for 18 small posterior pole uveal melanomas. Am J Ophthalmol. 2006;141:840-9.

11. Mueller AJ, Talies S, Schaller UC, et al. Stereotactic radiosurgery of large uveal melanomas with the gamma-knife. Ophthalmology. 2000;107:1381-7.
12. Haas A, Pinter O, Papaefthymiou G, et al. Incidence of radiation retinopathy after high-dosage single-fraction gamma knife radiosurgery for choroidal melanoma Ophthalmology. 2002;109:909-13.

13. Gragoudas ES, Goitein M, Koehler AM, et al. Proton irradiation of small choroidal malignant melanomas. Am J Ophthalmol. 1977;83:665-73.

14. Foss AJE, Whelehan I, Hungerford JL, et al. Predictive factors for the development of rubeosis following proton beam radiotherapy for uveal melanoma. Br J Ophthalmol. 1997;81:748-54.

15. Kincaid MC, Forberg R, Torczynski E, et al. Complications after proton beam therapy for uveal malignant melanoma. A clinical and histopathologic study of five cases. Ophthalmology. 1988;95:982-91.

16. Young LHY, Gragoudas ES. Macular uveal melanoma treated with proton beam irradiation. Retina. 1994;14:43-6.

17. Tokuuye K, Akine Y, Sumi M, et al. Fractionated stereotactic radiotherapy for choroidal melanoma. Radiother Oncol. 1997;43:87-91.

18. Zehetmayer M, Kitz K, Menapace R, et al. Local tumor control and morbidity after one to three fractions of stereotactic external beam irradiation for uveal melanoma. Radiother Oncol. 2000;55:135-44.

19. Wilkes SR, Gragoudas ES. Regression patterns of uveal melanomas after proton beam irradiation. Ophthalmology. 1982;89:840-4.

20. Emara K, Weisbrod DJ, Sahgal A, et al. Stereotactic radiotherapy in the treatment of juxtapapillary choroidal melanoma: preliminary results. Int J Radiat Oncol Biol Phys. 2004;59:94-100.

21. Conway RM, Poothullil AM, Daftari IK, et al. Estimates of ocular and visual retention following treatment of extra-large uveal melanomas by proton beam radiotherapy. Arch Ophthalmol. 2006;124:838-43.

22. Gragoudas ES, Goitein M, Koehler A, et al. Proton beam irradiation of choroidal melanomas. Arch Ophthalmol. 1978;96:1583-91.

23. Gragoudas ES, Seddon JM, Egan K . Long-term results of proton beam irradiated uveal melanomas. Ophthalmology. 1987;94:349-53.

24. Brovkina AF, Zarubei GD. Ciliochoroidal melanomas treated with a narrow medical proton beam. Arch Ophthalmol. 1986;104:402-4.

25. Gragoudas ES. Proton beam irradiation of uveal melanomas: the first 30 years. The Weisenfeld Lecture. Invest Ophthalmol Vis Sci. 2006;47:4666-73.

26. Kim MK, Char DH, Castro JL, et al. Neovascular glaucoma after helium ion irradiation for uveal melanoma. Ophthalmology. 1986;93:189-92.

27. Gragoudas ES, Lane AM. Uveal melanoma: proton beam irradiation. Ophthalmol Clin North Am. 2005;18:111-18.

28. Lee J, Logani S, Lakosha H, et al. Preretinal neovasculaisation associated with choroidal melanoma. Br J Ophthalmol. 2001;85:1309-12.

29. Egger E, Zografos L, Schalenbourg A, et al. Eye retention after proton beam radiotherapy for uveal melanoma. Int J Radiat Oncol Biol Phys. 2003;55:867-80.

30. Egan KM, Gragoudas ES, Seddon JM, et al. The risk of enucleation after proton beam irradiation of uveal melanoma. Ophthalmology. 1989;96:1377-82.

31. Dieckmann K, Georg D, Zehetmayer M, et al. LINAC based stereotactic radiotherapy of uveal melanoma: 4 years clinical experience. Radiother Oncol. 2003;67:199-206.

32. Robertson DM. A rationale for comparing radiation to enucleation in the management of choroidal melanoma. Am J Ophthalmol. 1989; 108:448-51.

33. Li. W, Gragoudas ES, Egan KM. Tumor basal area and metastatic death after proton beam irradiation for choroidal melanoma. Arch Ophthalmol. 2003;121:68-72.

34. Jaywant SM, Osei EK, Ladak S. Stereotactic radiotherapy in the treatment of ocular melanoma: A noninvasive eye fixation aid and tracking system. J Appl Clin Med Phys. 2003;4:156-61.

35. Constable IJ. Proton irradiation therapy for ocular melanoma. Trans Opthalmol Soc U K. 1977;97:430.

36. Gragoudas ES, Goitein M, Verhey L, et al. Proton beam irradiations of uveal melanomas. Arch Ophthalmol. 1982;100:928-34. 
